\title{
Patient Positining with Laser Based Body Surface Guided System and Intra-Fraction Motion Detection
}

\section{Lazer Tabanlı Vücut Yüzeyi Tarama Sistemi ile Hasta Pozisyonlaması ve Intra-fraction Hareket Takibi}

\author{
Bora Taş, İsmail Faruk Durmuş \\ Yeni Yüzyıl Üniversitesi Gaziosmanpaşa Hastanesi, Radyasyon Onkolojisi Bölümü, İstanbul
}

\section{ÖZET}

GíRiş ve AMAÇ: Sunulan çalışmada, vücut yüzeyi tarama sistemi kullanılarak akciğer ve meme kanseri hastalarının pozisyonlanması gerçekleştirildi. CBCT çekim sonuçları 12 hasta için 200 fraksiyon süresince karşılaştırıldı ve tedavi esnasında intra-fraction hasta hareketleri incelendi.

YÖNTEM ve GEREÇLER: $3.0 \mathrm{~mm}$ kesit aralıklı CT görüntüleri kullanılarak akciğer hastalarının radyoterapi tedavi planlaması VMAT, meme kanseri hastalarının ise IMRT yöntemi ile gerçekleştirildi. Referans görüntüler ile 200 fraksiyon süresince tedavi odasında hastaların pozisyonlanması C-RAD vücut yüzeyi tarama sistemi ile gerçekleştirilerek masa kaydırmaları yapıldı, ardından hastaların CBCT çekimleri gerçekleştirildi. Vücut yüzeyi takip sistemi ve CBCT sonucunda elde edilen korelasyon belirlendi. Tedavi esnasında hastaların vücut yüzeyi takip edilerek intra-fraction hareketleri her hasta için elde edildi.

BULGULAR: CBCT çekimi sonucunda elde edilen masa kaydırma değerleri 87 fraksiyonda 7 akciğer kanseri hastası için lateral yönde $0,22 \pm 0,08 \mathrm{~cm}$, longitidunial yönde $0,28 \pm 0,08 \mathrm{~cm}$ ve vertikal yönde $0,30 \pm 0,10 \mathrm{~cm}$ olarak elde edildi. 5 Meme kanseri hastası için 113 fraksiyonda karşılaştırma sonucunda lateral yönde $0,22 \pm 0,06$ $\mathrm{cm}$, longitidunial yönde $0,25 \pm 0,03 \mathrm{~cm}$ ve vertikal yönde $0,21 \pm 0,08 \mathrm{~cm}$ olarak elde edildi. Tedavi süresince 7 akciğger kanseri hastasında meydana gelen intra-fraction hareketi $0-1 \mathrm{dk}$. aralığında $0,7 \pm 0,4 \mathrm{~mm}, 1-2 \mathrm{dk}$. aralığında $1,5 \pm 0,5 \mathrm{~mm}, 2-3 \mathrm{dk}$. aralığında $2,4 \pm 0,7 \mathrm{~mm}, 3-4 \mathrm{dk}$. aralı̆̆ında $3,1 \pm 0,6 \mathrm{~mm}, 4-5 \mathrm{dk}$. aralığında $3,7 \pm 0,9 \mathrm{~mm}$ ve $5-6 \mathrm{dk}$. aralığında $4,1 \pm 1,4 \mathrm{~mm}$ olarak belirlendi.5 meme kanseri hastasında meydana gelen intra-fraction hareketi ise 0$1 \mathrm{dk}$. aralığında $0,6 \pm 0,1 \mathrm{~mm}, 1-2 \mathrm{dk}$. aralı̆̆ında $1,6 \pm 0,2 \mathrm{~mm}, 2-3 \mathrm{dk}$. aralığında $2,6 \pm 0,5 \mathrm{~mm}, 3-4 \mathrm{dk}$. aralığında $3,2 \pm 0,4 \mathrm{~mm}, 4-5 \mathrm{dk}$. aralığında $3,8 \pm 0,7 \mathrm{~mm}, 5-6 \mathrm{dk}$. aralığında $4,2 \pm 0,4 \mathrm{~mm}$ ve $6-7 \mathrm{dk}$. aralığında $4,4 \pm 0,7 \mathrm{~mm}$ olarak elde edildi.

TARTIŞMA ve SONUÇ: Akciğer ve meme kanseri hastalarının vücut yüzeyi tarama sistemi ile pozisyonlanması ve CBCT çekimi sonucu sonuçlarının 200 fraksiyon süresince karşılaştırılması ile ortalama $0,3 \mathrm{~cm}$ 'in altında farklılık elde edildi. Hastaların pozisyonlanması için iyonlaştıııcı radyasyonun kullanımının azaltılmasında vücut yüzeyi taraması sisteminin kullanımının akciğer ve meme kanseri radyoterapisinde uygun olduğu tespit edildi.

Anahtar Kelimeler: Meme Ca, VMAT, CBCT

\begin{abstract}
INTRODUCTION: In this study, we adjusted lung and breast cancer patient's positioning by using body surface scanning system. We compared CBCT scan's results deviation for 12 patients during 200 fractions and we investigated intra-fraction motion of patients.

METHODS: Lung cancer patient's radiotherapy treatment planning were performed with VMAT, breast cancer's patients with IMRT method while using 3,0 mm thickness CT slices. Couch shifts were performed while using C-RAD body surface scanning system during 200 fractions in the treatment room, then patient's CBCT scans were performed. We obtained body surface guided scanning system and CBCT scan correlation. Intrafraction motion was obtained during treatment for each patient while using body surface guided. RESULTS: Couch shift parameters were obtained in lateral direction $0,22 \pm 0,07 \mathrm{~cm}$, longitudinal direction $0,28 \pm 0,08 \mathrm{~cm}$ and vertical direction $0,30 \pm 0,10 \mathrm{~cm}$ during 87 fractions for 7 lung cancer patients. Correlation results were obtained in lateral direction $0,22 \pm 0,06 \mathrm{~cm}$, longitidunial direction $0,25 \pm 0,03 \mathrm{~cm}$ and vertical direction $0,21 \pm 0,08 \mathrm{~cm}$ during 113 fractions for 5 breast cancer patients. We determined intra-fraction motion
\end{abstract}


$0,7 \pm 0,4 \mathrm{~mm}$ in 0 - $1 \mathrm{~min}$. interval, $1,5 \pm 0,5 \mathrm{~mm}$ in $1-2 \mathrm{~min}$. interval, $2,4 \pm 0,7 \mathrm{~mm}$ in $2-3 \mathrm{~min}$. interval, $3,1 \pm 0,6 \mathrm{~mm}$ in 3 4min. interval, $3,8 \pm 0,7 \mathrm{~mm}$ in 4-5min. interval and 4,1 $\pm 1,4 \mathrm{~mm}$ in 5-6min. interval for 7 lung cancer patients. We obtained intra-fraction motion $0,6 \pm 0,1 \mathrm{~mm}$ in $0-1 \mathrm{~min}$. interval, $1,6 \pm 0,2 \mathrm{~mm}$ in $1-2 \mathrm{~min}$. interval, $2,6 \pm 0,5 \mathrm{~mm}$ in 2 $3 \mathrm{~min}$. interval, $3,2 \pm 0,4 \mathrm{~mm}$ in $3-4 \mathrm{~min}$. interval, $3,8 \pm 0,7 \mathrm{~mm}$ in $4-5 \mathrm{~min}$. interval, $4,2 \pm 0,4 \mathrm{~mm}$ in $5-6 \mathrm{~min}$. and $4,4 \pm 0,7 \mathrm{~mm}$ in $6-7 \mathrm{~min}$. interval for 7 lung cancer patients.

DISCUSSION AND CONCLUSION: Correlation between body surface guided system posioning and CBCT positioning were obtained an average less than $0.3 \mathrm{~cm}$ for lung and breast cancer patients during 200 fractions. Reducing the ionisation radiation for patient positioning with body surface guided system were obtained feasible for lung and breast cancer radiotherapy.

Keywords: Breast Ca, VMAT, CBCT

\section{GİRIŞ}

Radyoterapi'nin altın kuralı; tanımlanmış bir hedef hacme belirlenmiş ideal dozu verirken, çevredeki sağlıklı dokuya olabildiğince az radyasyon vererek kür şansını arttırırken iyi bir yaşam kalitesini de beraberinde sağlamaktır [1]. Radyoterapi, kanser tedavisinde X-1şınları, gama 1şınları, elektronlar ve yüklü parçacıklar gibi iyonize radyasyonun kullanıldığı bir tedavi yöntemidir [2,3]. Günümüzde bilimsel ve teknolojik gelişmeler ile tümörü tedavi ederken, normal dokuların radyasyonun olumsuz etkilerinden en iyi şekilde korunmasına olanak sağlamak amacıyla üç boyutlu konformal radyoterapi (3BKRT), görüntü eşliğinde radyoterapi (Image Guided Radiation Therapy, IGRT) olanağı sayesinde ise organ ve hasta hareketlerini de hesaba katarak, yoğunluk ayarlı radyoterapi (Intensity Modulated Radiation Therapy, YART/IMRT), VMAT (Volumetric Modulated Arc Therapy), SRS (Stereotactic Radiation Surgery), SBRT (Stereotactic Body Radiation Therapy) gibi ileri radyoterapi tedavi teknikleri uygulanmaktadır. Uniform 1 şın demetleri ile yapılan tedavilerde, özellikle konkav tümörlerin istenildiği gibi 1şınlanması 3BKRT ile pek mümkün olamamaktadır. Teknik olarak, tedavi planlarında her bir alan kendi içinde küçük alancıklara bölünerek ve radyasyon miktarları değiştirilerek ışınlandığında, uniform olmayan ışın demetleri elde edilir. Her bir alancık segment olarak isimlendirilir. Yoğunluk Ayarlı Radyoterapi, çok yapraklı kolimatörler kullanılarak, Step and Shoot ve Dynamic (sliding windows) olmak üzere iki farklı yöntemle yapılır. YART tedavisinde her iki işlem yapıllırken gantry ve kolimatör açısı sabittir. VMAT tedavi tekniğinde ise gantry açısı hareket halindeyken sliding windows tekniği ile çoklu yapraklar hareket ederek segmentler oluştururken ışınlama devam eder. Belirli gantry açı aralıklarında planlama doğrultusunda doz çıkışı çoklu yaprakların sürekli hareket halinde olarak çalışması ile meydana gelen tedaviye VMAT tedavisi denilir. Bu tedavi tam tur olabileceği gibi parsiyel olarak da uygulanabilir.

Meme kanserinde YART ve VMAT radyoterapi tekniği, tedavi edilen memede doz homojenitesini arttıran ve fiziksel kama filtre kullanılmamasından ötürü oluşan saçılma dozlarını azaltan bir yöntemdir aynı zamanda çevredeki normal dokularda toksisiteyi minimuma indirerek dozimetrik avantaj sağlamaktadır. $\mathrm{Bu}$ tekniklerden bir tanesi 3BKRT tekniğinde ki gibi tanjensiyel alanlar belirledikten sonra alt alanlar (field and field) oluşturup düz planlama tekniğiyle kama filtre kullanmaksızın homojen bir doz dağılımı elde edilmesi yöntemidir. Diğer bir teknik ise ters planlama algoritması kullanıp 5 ile 9 arasinda değişen alanlar belirlenerek yapılan tedavi tekniğidir. Son zamanlar da VMAT RT tedavi tekniğini kullanıp parsiyel alan veya alanlar yardımıyla meme kanserinin radyoterapi tedavisi mümkündür. Hurmanks ve ark.[4] normal doku komplikasyon olasılıkları (NTCP) modelinde kardiyak ve akciğer komplikasyonları göz önüne alındığında YART tedavisinde 3BKRT tedavisine göre kardiyak toksisitede azalma mevcutken, minimal bir etkinin akciğer için zatüre olasılığında mevcut olduğuna 
değinmiştir.Hong ve ark.[5] YART tedavisi ile dozimetrik olarak yayınladıkları raporda 10 meme kanseri vakasında önemli ölçüde kardiyak arterde, iki akciğer hacmi toplamında ve etrafindaki sağlam dokularda doz düşüşünü belirlemişlerdir.

Akciğer kanserinin radyoterapi ile tedavisinde birincil tümöre ve nodal metastazlara doz verilirken özofagus, kalp, omuirilik dozları ve doz alan akciğer hacmi de dahil olmak üzere çevre dokuların toksisiteleri dikkate alınmalıdır. Akciğer kanseri tedavisinde YART ve VMAT radyoterapi tedavi teknikleri yaygin olarak kullanilan teknikler olup sağlam organ dozlarını 3BKRT tekniğine göre ciddi oranda azaltmaktadır ve hedef organ doz konformalitesinde belirgin iyileşmeler gerçekleştirebilmektedir. Murshed ve ark. [6] çalışmalarında 41 hastaya 3BKRT tekniği ile 63Gy vermişler. YART tekniği ile planlama yapıldığında akciğerin $10 \mathrm{~Gy}$ ve 20Gy alan hacimlerinin aldığı dozlar azalmıştır. Aynı zamanda hedefin doz konformalitesi YART ile gelişmiş, kalp ve özofagus dozlarında da düşüş görülmüştür.

Hangi radyoterapi tedavi tekniği kullanılırsa kullanılsın, istenilen tedavi dozunun hedef hacme hassas ve doğru şekilde uygulanabilmesinde görüntü eşliğinde radyoterapinin (IGRT) büyük önemi vardır.

Çalışmamızda; iyonize olmayan lazer tabanlı vücut yüzeyi tarama sisteminin akciğer ve meme kanseri hastalarının tedavi odasında pozisyonlamasinda ve tedavi esnasinda oluşabilecek hareketlerin takibinde kullanılmasının uygunluğu incelenmektedir.

\section{GEREÇ ve YÖNTEM}

Siemens Biograph mCT $^{\circledR}$ (Siemens, Germany) cihazında supin pozisyonda akciğer board'u ve meme board'u ile hastalar pozisyonlanarak 3,0 $\mathrm{mm}$ kesit aralıklı CT görüntüleri elde edildi ve akciğer kanseri hastalarının radyoterapi tedavi planlaması VMAT, meme kanser hastalarının ise YART yöntemi ile gerçekleştirildi. Lazer tabanlı vücut yüzeyi tarama sistemi Sentinel ${ }^{\circledR}$ ve Catalyst ${ }^{\circledR}$ (C-RAD, AB, Uppsala, Sweden) sistemleri $(\lambda=450 \mathrm{~nm})$ kullanılarak hastaların pozisyonlanmaları ve intra-fraction hareketleri gözlemlendi. Hastaların vücut yüzeyi dış konturlar1 Monaco $^{\circledR} 5.11$ tedavi planlama sisteminden C-RAD server sistemine gönderilerek hasta pozisyonlanması için referans görüntüler her hasta için elde edildi. Sentinel $^{\circledR}$ ve Catalyst $^{\circledR}$ vücut yüzeyi tarama sistemine gönderilmiş referans görüntüler ile hastaların tedavi öncesinde 200 fraksiyon süresince Versa $\mathrm{HD}{ }^{\circledR}$ lineer hizlandırıcı (Elekta AB, Stockholm, Sweden) tedavi cihazında pozisyonlanması Catalyst ${ }^{\circledR}$ vücut yüzeyi tarama sistemi ile gerçekleştirilerek gerekli masa kaydırmaları yapıldı (Resim 1.), ardından hastaların XVI ${ }^{\circledR}$ 5.0 IGRT sistemi ile CBCT çekimleri gerçekleştirildi. Vücut yüzeyi takip sistemi ve CBCT sonucunda elde edilen korelasyon 200 fraksiyon süresince akciğer ve meme kanseri hastaları için belirlendi. Tedavi esnasında hastaların vücut yüzeyi takip edilerek oluşan ortalama bağıl intra-fraction hareketleri her hasta için elde edildi.

\section{BULGULAR}

$\mathrm{XVI}^{\circledR} \quad$ 5.0 IGRT sisteminde CBCT çekimi sonucunda elde edilen masa kaydırma değerleri 87 fraksiyonda 7 akciğer kanseri hastası için lateral yönde $0,22 \pm 0,06 \mathrm{~cm}$, longitudinal yönde $0,28 \pm 0,08 \mathrm{~cm}$ ve vertikal yönde $0,30 \pm 0,10 \mathrm{~cm}$ olarak elde edildi. Hasta pozisyonlama değerleri Tablo 1.'de mevcuttur.

5 Meme kanseri hastası için 113 fraksiyonda bu karşılaştırma sonucunda lateral yönde $0,22 \pm 0,06 \mathrm{~cm}$, longitidunial yönde $0,25 \pm 0,03 \mathrm{~cm}$ ve vertikal yönde $0,21 \pm 0,08 \mathrm{~cm}$ olarak elde edildi. Hasta pozisyonlama değerleri Tablo 2.'de mevcuttur.

Tedavi süresince 7 akciğer kanseri hastasında meydana gelen intra-fraction hareketi $0-1 \mathrm{dk}$ aralığında $0,70 \pm 0,4 \mathrm{~mm}, 1-2 \mathrm{dk}$ aralığında

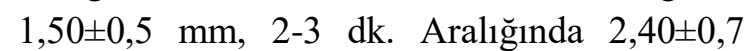
$\mathrm{mm}, 3-4 \mathrm{dk}$. aralı̆̆ında $3,10 \pm 0,6 \mathrm{~mm}, 4-5 \mathrm{dk}$. 
aralığında $3,70 \pm 0,9 \mathrm{~mm}$ ve 5-6 dk. aralığında $4,10 \pm 1,4 \mathrm{~mm}$ olarak belirlendi. Akciğer kanseri hastalarının ortalama bağıl hareket değerleri Tablo 3.'de mevcuttur.

5 meme kanseri hastasında meydana gelen intra-fraction hareketi ise $0-1 \mathrm{dk}$ aralığında $0,55 \pm 0,1 \mathrm{~mm}, 1-2 \mathrm{dk}$ aralığında

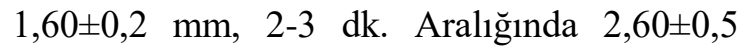

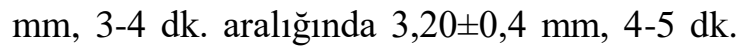
aralığında $3,80 \pm 0,7 \mathrm{~mm}, 5-6 \mathrm{dk}$. aralığında $4,20 \pm 0,4 \mathrm{~mm}$ ve $6-7 \mathrm{dk}$. aralığında $4,40 \pm 0,7$ $\mathrm{mm}$ olarak belirlendi. Meme kanseri hastalarının ortalama bağıl hareket değerleri Tablo 4.'de mevcuttur.

\section{TARTIŞMA}

Akciğer ve meme kanseri hastalarına iyonlaştırıcı radyasyon dozu vermeksizin Sentinel $^{\circledR}$ ve Catalyst ${ }^{\circledR}$ vücut yüzeyi taraması sistemi ile pozisyonlanması ile $\mathrm{XVI}^{\circledR} \quad 5.0$ CBCT pozisyonlanmasının karşılaştırılması sonucunda 200 fraksiyonda ortalama lateral yönde $0,22 \pm 0,07 \mathrm{~cm}$, longitidunial yönde
$0,27 \pm 0,06 \mathrm{~cm}$ ve vertikal yönde $0,25 \pm 0,10 \mathrm{~cm}$ olarak elde edildi. Michalski A. ve ark.[7] meme kanseri hastalarının radyoterapisinde 5,0 mm' nin altında intra-fraction hareketleri belirlemişdir ve sonuçlar bizim çalışmamız ile uyumludur. Li ve ark. [8] akciğer kanseri SBRT tedavisinde CBCT çekimi ile intrafraction hareketini 4,0 $\mathrm{mm}$ olarak tespit etmişlerdir, SBRT tedavisinde uygulanan yüksek dozlar tedavi süresini arttırmakta ve bu durum hasta hareketlerinin de artışına neden olabilmektedir, çalışmamızda elde ettiğimiz sonuçlar ile uyumludur. Hastaların pozisyonlanması için iyonlaştırıcı radyasyonun kullanıminın azaltılmasında Sentinel $^{\circledR}$ ve Catalyst $^{\circledR}$ vücut yüzeyi taraması sisteminin kullanımının uygun olduğu tespit edildi. Hastaların intra-fraction hareketi göz önünde bulundurularak gerekli marjlar verilmesinde ve hareketin verilen marjları aştı̆̆ 1 durumlarda vücut yüzeyi tarama sistemi ve/veya CBCT sistemi ile hastanın tekrar pozisyonlanması önerilmektedir.

Table 1. Akciğer CA hastalarında CBCT - Vücut Yüzeyi Takip Sistemi Korelasyonu

\begin{tabular}{ccccc} 
Hasta & Fraksiyon & $\begin{array}{c}\text { Lateral yönde } \\
(\mathbf{c m})\end{array}$ & $\begin{array}{c}\text { Longitidunal } \\
\text { yönde }(\mathbf{c m})\end{array}$ & $\begin{array}{c}\text { Vertical yönde } \\
(\mathbf{c m})\end{array}$ \\
\hline 1 & 10 & 0,38 & 0,20 & 0,24 \\
2 & 11 & 0,24 & 0,27 & 0,30 \\
3 & 14 & 0,18 & 0,40 & 0,48 \\
4 & 5 & 0,13 & 0,14 & 0,39 \\
5 & 12 & 0,24 & 0,29 & 0,20 \\
6 & 16 & 0,18 & 0,31 & 0,25 \\
7 & 19 & 0,20 & 0,26 & 0,29 \\
\hline Ort. \pm SD & $\mathbf{8 7}$ & $\mathbf{0 , 2 2 \pm 0 , 0 8}$ & $\mathbf{0 , 2 8 \pm 0 , 0 8}$ & $\mathbf{0 , 3 0 \pm 0 , 1 0}$
\end{tabular}


Tablo 2. Meme CA hastalarında CBCT - Vücut Yüzeyi Takip Sistemi Korelasyonu

\begin{tabular}{|c|c|c|c|c|}
\hline Hasta & Fraksiyon & $\begin{array}{l}\text { Lateral yönde } \\
\qquad(\mathrm{cm})\end{array}$ & $\begin{array}{l}\text { Longitidunal } \\
\text { yönde (cm) }\end{array}$ & $\begin{array}{l}\text { Vertical yönde } \\
\text { (cm) }\end{array}$ \\
\hline 1 & 18 & 0,29 & 0,26 & 0,33 \\
\hline 2 & 23 & 0,16 & 0,21 & 0,19 \\
\hline 3 & 22 & 0,16 & 0,29 & 0,11 \\
\hline 4 & 28 & 0,29 & 0,28 & 0,25 \\
\hline 5 & 22 & 0,22 & 0,23 & 0,21 \\
\hline Ort. $\pm S D$ & 113 & $0,22 \pm 0,06$ & $0,25 \pm 0,03$ & $0,21 \pm 0,08$ \\
\hline
\end{tabular}

Tablo 3. Akciğer CA hastalarında Intra-fraction ortalama bağıl hareket değerleri

\begin{tabular}{|c|c|c|c|c|c|c|}
\hline \multirow[b]{2}{*}{ Hasta } & \multicolumn{6}{|c|}{ Ortalama Bağıl Hareket (mm) } \\
\hline & $\begin{array}{c}\text { 0-1 dk. } \\
\text { Süresince }\end{array}$ & $\begin{array}{c}\text { 1-2 dk. } \\
\text { süresince }\end{array}$ & $\begin{array}{l}\text { 2-3 dk. } \\
\text { süresince }\end{array}$ & $\begin{array}{l}\text { 3-4 dk. } \\
\text { süresince }\end{array}$ & $\begin{array}{l}\text { 4-5 dk. } \\
\text { süresince }\end{array}$ & $\begin{array}{l}\text { 5-6 dk. } \\
\text { süresince }\end{array}$ \\
\hline 1 & 0,5 & 1,5 & 2,5 & 3,5 & 4,0 & - \\
\hline 2 & 0,5 & 1,5 & 2,0 & 2,5 & 3,0 & 3,5 \\
\hline 3 & 0,5 & 0,5 & 1,0 & 2,0 & 2,0 & 2,5 \\
\hline 4 & 1,5 & 2,0 & 3,5 & 4,0 & 4,5 & 5,5 \\
\hline 5 & 1,0 & 2,0 & 2,5 & 3,5 & 4,5 & 5,0 \\
\hline 6 & 0,5 & 1,5 & 2,5 & 3,0 & 3,5 & - \\
\hline 7 & 0,5 & 1,5 & 2,5 & 3,5 & 4,5 & - \\
\hline Ort. $\pm S D$ & $0,7 \pm 0,4$ & $1,5 \pm 0,5$ & $2,4 \pm 0,7$ & $3,1 \pm 0,6$ & $3,7 \pm 0,9$ & $4,1 \pm 1,4$ \\
\hline
\end{tabular}

Tablo 4. Meme CA hastalarında Intra-fraction ortalama bağıl hareket değerleri Ortalama Bağıl Hareket (mm)

\begin{tabular}{|c|c|c|c|c|c|c|c|}
\hline Hasta & $\begin{array}{c}\text { 0-1 dk. } \\
\text { Süresince }\end{array}$ & $\begin{array}{c}1-2 \mathrm{dk} . \\
\text { süresince }\end{array}$ & $\begin{array}{c}\text { 2-3 dk. } \\
\text { süresince }\end{array}$ & $\begin{array}{c}\text { 3-4 dk. } \\
\text { süresince }\end{array}$ & $\begin{array}{c}\text { 4-5 dk. } \\
\text { süresince }\end{array}$ & $\begin{array}{c}\text { 5-6 dk. } \\
\text { süresince }\end{array}$ & $\begin{array}{l}\text { 6-7 dk. } \\
\text { süresince }\end{array}$ \\
\hline 1 & 0,5 & 2,0 & 3,5 & 3,5 & 4,5 & 4,5 & 4,5 \\
\hline 2 & 0,5 & 1,5 & 2,5 & 3,5 & 4,5 & 4,5 & 5,5 \\
\hline 3 & 0,5 & 1,5 & 2,0 & 2,5 & 3,0 & 3,5 & 3,5 \\
\hline 4 & 0,5 & 1,5 & 2,5 & 3,5 & 3,5 & 4,5 & 4,5 \\
\hline 5 & 0,8 & 1,5 & 2,5 & 3,0 & 3,5 & 4,0 & 4,0 \\
\hline Ort. $\pm S D$ & $0,6 \pm 0,1$ & $1,6 \pm 0,2$ & $2,6 \pm 0,5$ & $3,2 \pm 0,4$ & $3,8 \pm 0,7$ & $4,2 \pm 0,4$ & $4,4 \pm 0,7$ \\
\hline
\end{tabular}



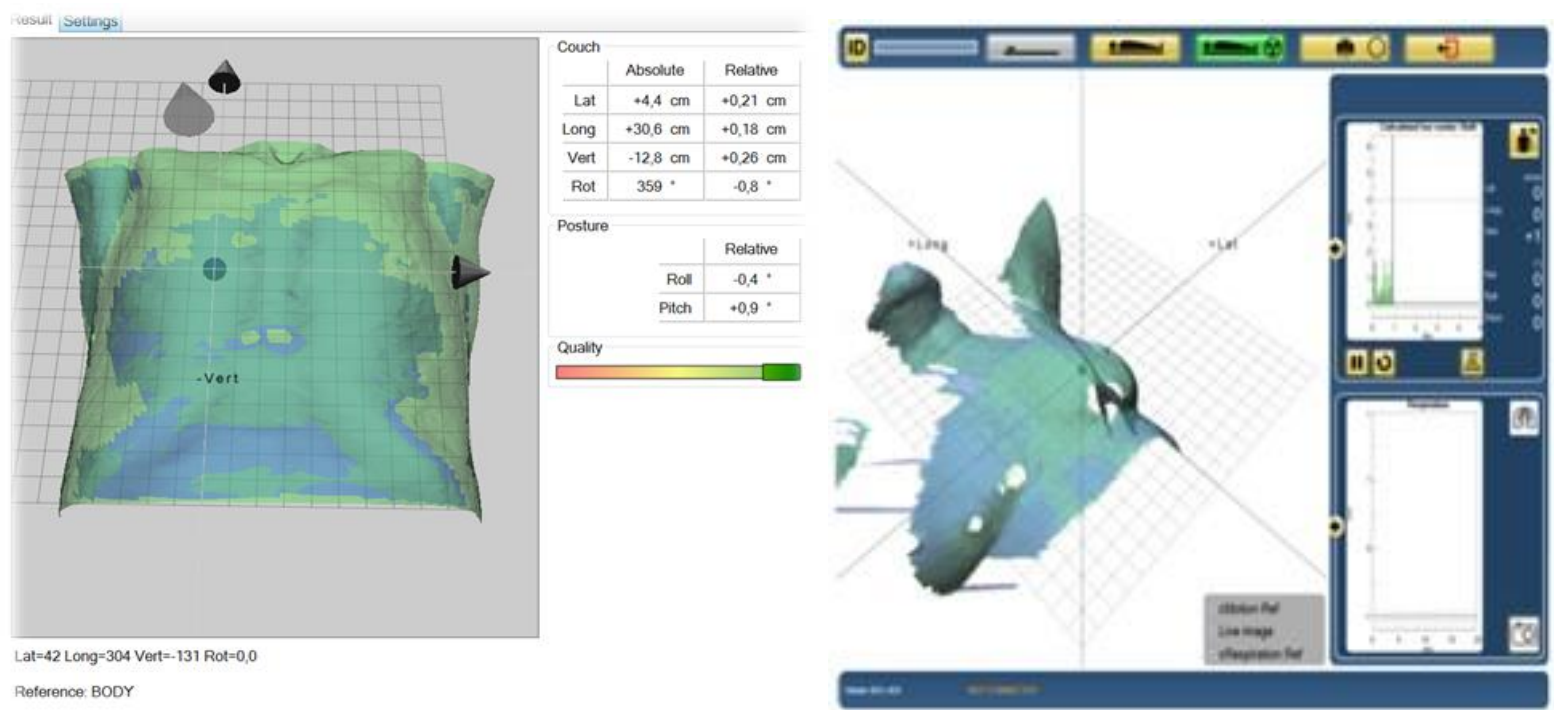

Resim 1: C-RAD Vücut Yüzeyi Takip Sistemi ile a) Meme Kanseri Hastası Pozisyonlanması b) Akciğer Kanseri Hastası Pozisyonlanması

\section{REFERENCES}

1. Pussey WA., (1903), "The Pratical Application of the Rontgen Rays in Therapeutics and Diagnosis," WB Sounders Comp.

2. Perez CA., Brady L.W., Roti J. L. R.., (1997), "Principles and Practices of Radiation Oncology", (3), 1.

3. Perez CA, Brady LW., (1992), "Principles and Practice of Radiation Oncology", Philadelphia, Lippincott Company, (2), 595-606.

4. Iuchi T, Hatano $\mathrm{K}$, Narita $\mathrm{Y}$, et al. Hypofractionated high-dose irradiation for the treatment of malignant astrocytomas using simultaneous integrated boost technique by IMRT. Int J Radiat Oncol Biol Phys Vol. 64. 2006. p.1317-1324.

5. Huang E, Teh BS, Strother DR, et al. Intensitymodulated radiation therapy for pediatric medulloblastoma: Early report on the reduction of ototoxicity. Int J Radiat Oncol Biol Phys Vol. 55. 2002. p.599-605.

6. Mutic S, Low DA, Klein EE, et al. Room shielding for intensity-modulated radiation therapy treatment facilities. Int J Radiat Oncol Biol Phys. Vol.50. 2001. p.239-246.

7. Michalski A, Atyeo J, Cox J et al. Inter- and intra-fraction motion during radiation therapy to the whole breast in the supine position: a systematic review. J Med Imaging Radiat Oncol. Vol. 56. 2012. p.499-509.

8. Li W, Purdie TG, Taremi M et al. Effect of immobilization and performance status on intrafraction motion for stereotactic lung radiotherapy: analysis of 133 patients. Int J Radiat Oncol Biol Phys. Vol. 81. 2011. p.156875 . 